\title{
Scaling Analysis of Improved Actions for Pure SU(3) Gauge Theory *
}

\author{
QCD-TARO : Ph. de Forcrand ${ }^{a}$, M. García Peréz ${ }^{\mathrm{b}}$, T. Hashimoto ${ }^{\mathrm{c}}$, S. Hioki ${ }^{\mathrm{d}}$, H. Matsufuru ${ }^{\mathrm{e}, \mathrm{f}}$, \\ O. Miyamura ${ }^{\text {, A. Nakamura }}{ }^{f}$, I.-O Stamatescu ${ }^{f, g}$, T. Takaishi ${ }^{\text {h }}$ and T. Umeda ${ }^{e}$
}

asCSC, ETH-Zürich, CH-8092 Zürich, Switzerland

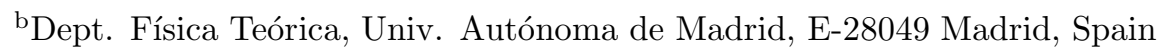

${ }^{\text {c} D e p t . ~ o f ~ A p p l . ~ P h y s ., ~ F a c . ~ o f ~ E n g i n e e r i n g, ~ F u k u i ~ U n i v ., ~ F u k u i ~ 910-8507, ~ J a p a n ~}$

${ }^{\mathrm{d} D e p t . ~ o f ~ P h y s i c s, ~ T e z u k a y a m a ~ U n i v ., ~ N a r a ~ 631-8501, ~ J a p a n ~}$

${ }^{\text {e}}$ Dept. of Physics, Hiroshima Univ., Higashi-Hiroshima 739-8526, Japan

${ }^{\mathrm{f}}$ Res. Inst. for Inform. Sci. and Education, Hiroshima Univ., Higashi-Hiroshima 739-8521, Japan

gFEST, Schmeilweg 5, D-69118 Heidelberg, Germany

${ }^{\mathrm{h}}$ Hiroshima University of Economics, Hiroshima 731-01, Japan

We have explored the behaviour of some improved actions based on a nonperturbative renormalization group $(\mathrm{RG})$ analysis in coupling space. We calculate the RG flow in two-coupling space $\left(\beta_{1 \times 1}, \beta_{1 \times 2}\right)$ and examine the restoration of rotational invariance and the scaling of physical quantities $\left(T_{c} / \sqrt{\sigma}\right)$.

\section{Introduction}

Many improved actions have been proposed and studied which may allow us much more reliable simulations near the continuum limit within the limited computer resources existing nowadays. One way to obtain such improved actions is to find the renormalized trajectory (RT), any point on which serves as a "perfect action" 13]. Here we will consider the simplest case, i.e., actions in two coupling $\left(\beta_{1 \times 1}, \beta_{1 \times 2}\right)$ space, which for practical purposes are preferable than actions with a larger number of operators. In this contribution, we evaluate the RG flow and study whether the performance of such actions improves when they approach the renormalized trajectory.

\section{Search of the Renormalized Trajectory}

*Talks by T. Umeda at LATTICE98

\subsection{MCRG}

We adapt Swendsen's blocking transformation scheme [6], which is defined as

$$
\begin{gathered}
P(U)=U_{\mu}(x) U_{\mu}(x+\mu) \\
+\frac{1}{2} \sum_{\nu \neq \mu} U_{\nu}(x) U_{\mu}(x+\nu) U_{\mu}(x+\nu+\mu) U_{\nu}^{\dagger}(x+2 \mu)
\end{gathered}
$$

Under this transformation the lattice spacing is doubled. Repeating this blocking transformation, we can get an action near the RT.

After each transformation we generate a blocked configuration, the couplings on which can be determined via the Schwinger-Dyson equation:

$$
\begin{gathered}
\frac{8}{3} \operatorname{Re}\left\langle\operatorname{Tr}\left(U_{l} G_{l}^{\alpha}\right)\right\rangle=\sum_{\gamma} \frac{\beta_{\gamma}}{6}\left\{-\operatorname{Re}\left\langle\operatorname{Tr}\left(U_{l} G_{l}^{\alpha} U_{l} G_{l}^{\gamma}\right)\right\rangle(2)\right. \\
\left.+\operatorname{Re}\left\langle\operatorname{Tr}\left(G_{l}^{\alpha}\left(G_{l}^{\gamma}\right)^{\dagger}\right)\right\rangle-\frac{2}{3}\left\langle\operatorname{Im} \operatorname{Tr}\left(U_{l} G_{l}^{\alpha}\right) \operatorname{Im} \operatorname{Tr}\left(U_{l} G_{l}^{\gamma}\right)\right\rangle\right\}
\end{gathered}
$$

where $G_{l}^{\gamma}$ are the "staples" corresponding to the link $U_{l}$. Solving these equations, we can calculate the coefficients of operators corresponding to the blocked action. In this paper we will ne- 
glect truncation errors and assume that the resulting blocked actions also have only two couplings, $\beta_{1 \times 1}$ and $\beta_{1 \times 2}$.

Figure 11 shows the blocking transformation flow in two-coupling space.

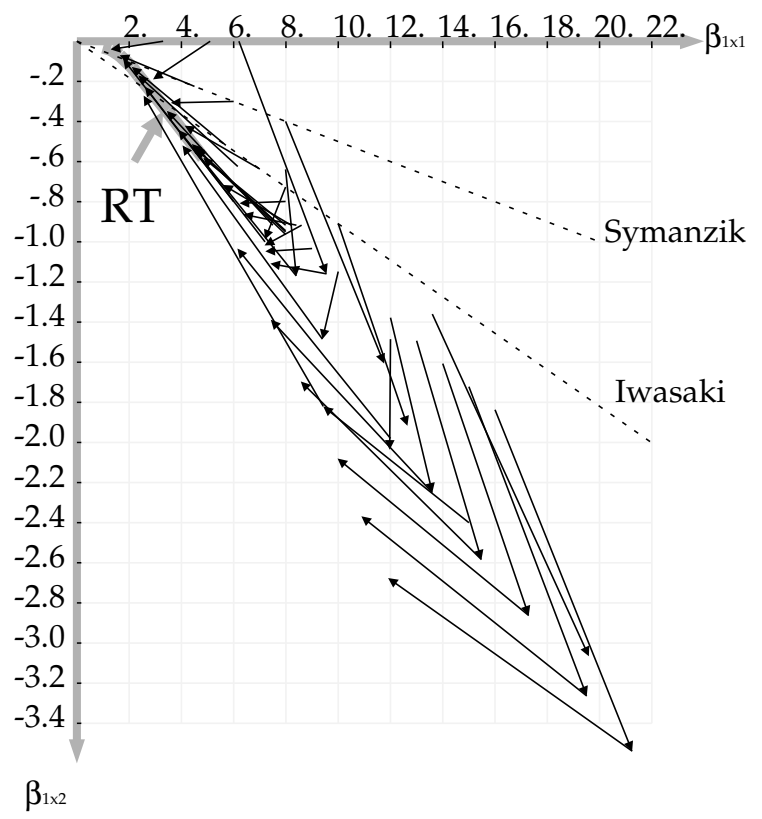

Figure 1. Blocking transformation flow in 2 coupling space

\subsection{Strong coupling limit}

The RT can be calculated within the strong coupling expansion. The string tension is obtained from the expectation value of Wilson loops. To leading order,

$\sigma a^{2} \simeq-\ln \frac{\left\langle W_{1 \times 2}\right\rangle}{\left\langle W_{1 \times 1}\right\rangle} \simeq-\ln \left(\frac{\beta_{1 \times 1}}{18}+\frac{\beta_{1 \times 2}}{\beta_{1 \times 1}}\right)$

Imposing that in the strong coupling limit, the lattice spacing goes to infinity, one easily derives the following relation

$\frac{\beta_{1 \times 1}}{18}+\frac{\beta_{1 \times 2}}{\beta_{1 \times 1}} \rightarrow 0$ with $a \rightarrow \infty$
As this equation tells us, in this limit, the RT is a parabola which is presented in Figure 11 to compare with the RT obtained by solving the S$\mathrm{D}$ equations as indicated above.

One could as well solve the S-D equations with coefficients calculated by the strong coupling expansion. This is work in progress and the results will be reported elsewhere.

\section{Scaling analysis}

Here we report on the scaling analysis of several improved actions. We study the violation of rotational symmetry as well as $T_{c} / \sqrt{\sigma}$.

\subsection{Violation of rotational symmetry}

First we define the following quantity which represents the violation of rotational symmetry

$\delta_{V}^{2} \equiv \sum_{\text {off }} \frac{\left[V(R)-V_{\text {on }}(R)\right]^{2}}{V(R)^{2} \delta V(R)^{2}}\left(\sum_{\text {off }} \frac{1}{\delta V(R)^{2}}\right)^{-1}$

where $V(R)$ is the static quark potential and $\delta V(R)$ its error. $V_{o n}(R)$ is a fitting function from only on-axis data, and $\sum_{\text {off implies }}$ summation over only off-axis data. We calculate this quantity with various improved actions. We write $\left(\beta_{1 \times 1}, \beta_{1 \times 2}\right)=\beta\left(c_{1 \times 1}, c_{1 \times 2}\right)$, and impose the condition, $c_{1 \times 1}+8 c_{1 \times 2}=$ 1. The Wilson action corresponds $c_{1 \times 2}=0$. For Symanzik(tree), Iwasaki and DBW2 actions, $c_{1 \times 2}=-1 / 12,-0.331,-1.4088$, respectively 伍, 2]. DBW2 (Double Blocked from Wilson action in 2 coupling space) is defined in ref. [8]. Results are summarized in Figure 2.

Simulations have been performed on a lattice of size $12^{3} \times 24$. Thermalization is 5000 sweeps, while the interval between Wilson loop measurements is 500 sweeps. We used 100 configurations.

\section{2. $T_{c} / \sqrt{\sigma}$}

Here we study the scaling behavior of $T_{c} / \sqrt{\sigma}$ for DBW2. The critical temperature is defined by

$T_{c}=1 / N_{t} a_{c}: a_{c}=a\left(\beta_{c}\right)$

where $N_{t}$ is the temporal extension of the lattice, and $a_{c}$ is the lattice spacing at critical cou- 


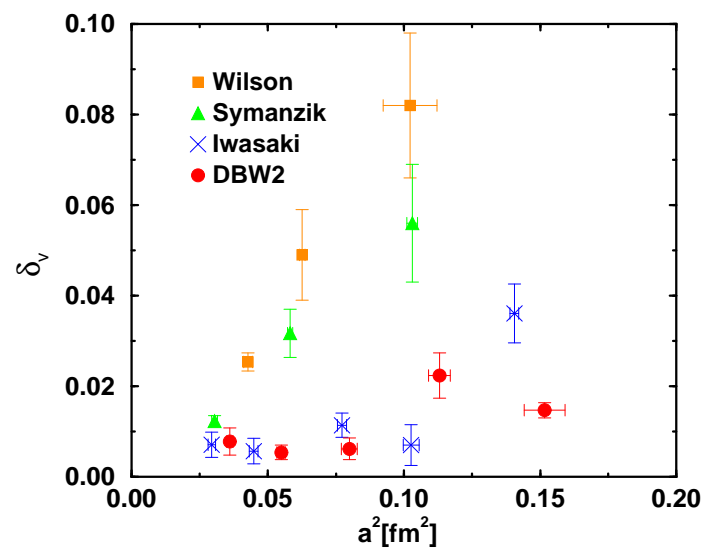

Figure 2. Rotational symmetry violation of various improved actions

pling $\beta_{c}$. In order to extract $\beta_{c}$, we calculate the Polyakov loop susceptibility on $N_{t}=4,6$ lattices. Then $\beta_{c}^{\prime} s$ in the infinite volume limit are obtained by using finite size scaling (of $N_{t}=4$ ). The results of $\beta_{c}$ for DBW2 are presented below.

\begin{tabular}{c|c|c}
\hline \hline & $N_{t}=4$ & $N_{t}=6$ \\
\hline lattice size & $12^{3} \times 4$ & $18^{3} \times 6$ \\
& $16^{3} \times 4$ & \\
\hline$\beta_{c}(\infty$ volume $)$ & $0.8243(95)$ & $0.936(25)$ \\
\hline \hline
\end{tabular}

Next we extract the string tension from the static quark potential at each value of $\beta_{c}$. For DBW2 the results for the static quark potential are

\begin{tabular}{c|c|c|c}
\hline \hline$\beta\left(N_{t}\right)$ & $A$ & $\alpha$ & $\sigma$ \\
\hline$\beta_{c}(4)$ & $0.550(17)$ & $-0.255(23)$ & $0.1555(28)$ \\
$\beta_{c}(6)$ & $0.579(96)$ & $-0.357(22)$ & $0.06996(99)$ \\
\hline \hline
\end{tabular}

where we used the Ansatz $V(R)=A+\alpha / R+\sigma R$.

For this action $T_{c} / \sqrt{\sigma}$ is $0.6340(60)$ and $0.6301(65)$ at $N_{t}=4$ and 6 respectively - see Fig. 3 for a comparison with other actions in twocoupling space.

\section{Summary}

We have studied the non-perturbative RT of $\mathrm{SU}(3)$ gauge theory in two-coupling space. We find an improvement of rotational invariance as the improved action becomes closer to the RT.

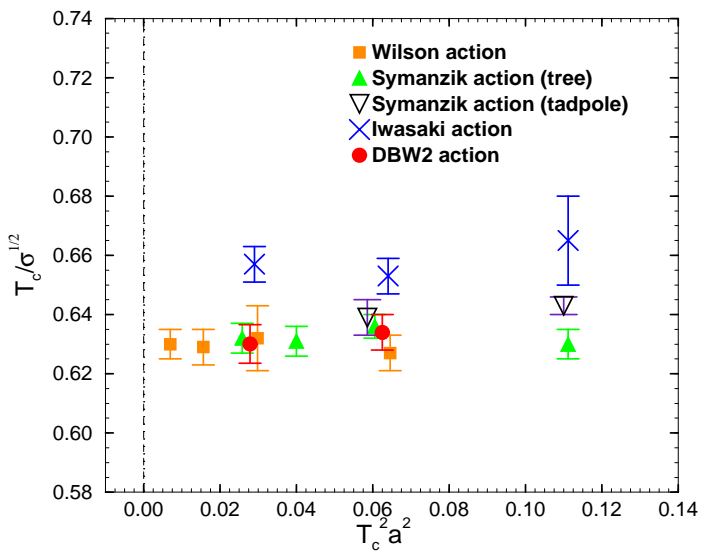

Figure 3. Scaling behavior of $T_{c} / \sqrt{\sigma}$. We compare DBW2 with results from other actions in 2-coupling space [9,10].

\section{REFERENCES}

1. K.G. Wilson, in Recent Developments in Gauge theories, ed. G. t'Hooft (Plenum Press, New York, 1980) p.363.

2. Y. Iwasaki, University of Tsukuba preprint, UTHEP-118, 1983.

3. P. Hasenfratz, F. Niedermayer, Nucl. Phys. B414 (1994) 785.

4. QCDTARO Collaboration, Phys. Rev. Lett., (1993) 71, 3963.

5. A. González-Arroyo and M. Okawa, Phys. Rev. D35 (1987) 672; Phys. Rev. B35 (1987) 2108.

6. R.H. Swendsen, Phys. Rev. Lett. 42 (1979) 859.

7. K. Symanzik, Nucl. Phys. B226 (1983) 187, 205

8. T. Takaishi, Phys. Rev. D54 (1996) 1050.

9. Y. Iwasaki and K. Kanaya, K. Kaneko, T.Yoshié, Phys.Rev.D 56 (1997) 151.

10. B. Beinlich, F. Karsch, E. Laermann and A. Peikert hep-lat/9707023 